The pattern of an abnormal median-normal sural (AMNS) sensory response is associated with acute and chronic inflammatory demyelinating polyradiculoneuropathy (AIDP and CIDP) and considered unusual in other types of neuropathy, although specificity and sensitivity of this pattern have not been evaluated. We compared sensory responses (patterns and absolute values) in patients with AIDP, CIDP, diabetic polyneuropathy (DP), and motor neuron disease (MND). Using strict criteria, the AMNS pattern occurred more frequently in recent onset AIDP $(39 \%)$ compared with CIDP $(28 \%)$, DP $(14 \%-23 \%)$, or MND $(22 \%)$ patients. This pattern was found in $3 \%$ of control subjects. The extreme pattern of an absent median-present sural response occurred only in AIDP and CIDP patients and in no other groups. Abnormalities of both nerves were more common in long-standing polyneuropathies such as CIDP and DP compared with AIDP or MND. Median nerve amplitudes were reduced significantly in AIDP, CIDP, and DP patients compared with MND patients, whereas sural nerve amplitudes were significantly reduced only in DP and CIDP patients. These findings may reflect early distal nerve involvement particularly in AIDP patients which is highlighted by differences in median and sural nerve recording electrode placement. We conclude that, in the appropriate clinical setting, the AMNS pattern, an absent median-present sural response pattern, or a reduced median amplitude compared with the sural amplitude supports a diagnosis of a primary demyelinating polyneuropathy. (C) 1993 John Wiley \& Sons, Inc.

Key words: sensory nerve conduction - axonal and demyelinating polyneuropathy

MUSCLE \& NERVE 16:262-266 1993

\title{
PATTERNS OF SENSORY NERVE CONDUCTION ABNORMALITIES IN DEMYELINATING AND AXONAL PERIPHERAL NERVE DISORDERS
}

\author{
MARK B. BROMBERG, MD, PhD, and JAMES W. ALBERS, MD, PhD
}

The pattern of nerve conduction abnormalities is important in the diagnosis of disorders of peripheral nerve. (One question that can be addressed by nerve conduction studies is whether there is electrodiagnostic evidence to distinguish between primary demyelinating and primary axonal polyneuropathy. "' Expected conduction changes for motor nerves have been reported for these two categories of polyneuropathy." Although similar nerve conduction changes are predicted for sen-

From the Departinent of Neurology, University of Michigan Medical Center, Ann Arbor. Michigan

Presented in part at the 38th annual meeting of the American Association of Electrodiagnostic Medicine, Vancouver, Canada, September 25-28, 1991

Address reprint requests to Dr. M.B. Bromberg, Department of Neurology, University of Michigan Medical Center, Taubman Center 1920/0316, 1500 East Medical Center Drive, Ann Arbor, Ml 48109-0316.

Accepted for publication July 2, 1992

CCC 0148-639X/93/030262-05

(c) 1993 John Wiley \& Sons. Inc sory nerves, the low amplitude and short duration of the sensory nerve action potential makes assessment of these changes difficult. ${ }^{15,17}$

Despite these limitations, the sensory nerve conduction pattern of an abnormal median-normal sural (AMNS) nerve response has been observed in both acute inflammatory demyelinating polyradiculoneuropathy (AIDP) ${ }^{1,10,20}$ and chronic inflammatory demyelinating polyradiculoneuropathy (CIDP), ${ }^{7}$ but is thought to occur rarely in axonal disorders. The diagnostic specificity and sensitivity of this pattern of sensory nerve responses has not been studied. We carried out a retrospective review of sensory nerve conduction data to compare findings in patients with disorders primarily affecting myelin to those primarily affecting the axon. Examples of primary demyelinating polyneuropathy included patients with AIDP and CIDP. Patients with diabetic polyneuropathy (DP) were used as a positive control group because, although they have a primary axonal polyneuropathy, they have demyelination to a minor degree and conduction slowing 
attributed to metabolic factors. ${ }^{8}$ Patients with amyotrophic lateral sclerosis-type motor neuron disease (MND) were used as a negative control group because the disease is a motor neuronopathy with minimal sensory nerve involvement. ${ }^{5,13}$

\section{METHODS}

The two patient groups with primary demyelinating polyneuropathy included 36 patients with AIDP and 64 patients with CIDP who received care in our Neuromuscular Clinic and met clinical criteria for the respective disorders. ${ }^{1,12} \mathrm{~Pa}$ tients with DP were divided into two groups. A DP-study group consisted of 30 patients with insulin-dependent and non-insulin-dependent diabetes mellitus and mild symptoms and signs of polyneuropathy who had baseline nerve conduction measurements as part of a therapeutic drug trial. A DP-consecutive group consisted of 29 consecutive patients with insulin-dependent or non-insulin-dependent diabetes referred to the EMG Laboratory for evaluation of polyneuropathy. The MND group consisted of 22 patients from our Neuromuscular Clinic who met clinical criteria for sporadic MND. ${ }^{5}$

Median and ulnar sensory nerve responses were recorded antidromically with ring electrodes on the second and fifth digits, respectively, following surface stimulation $14 \mathrm{~cm}$ proximally at the wrist and at the elbow. ${ }^{16}$ Sural nerve potentials were recorded with disc electrodes at the ankle following stimulation $14 \mathrm{~cm}$ proximally at the calf. ${ }^{16}$ Limb temperature was maintained above $32^{\circ} \mathrm{C}$ with application of hot packs. A sensory response was considered abnormal if any one of the values for distal amplitude, distal peak latency, or conduction velocity were beyond the EMG Laboratory normal limits (see Table 2). Normal laboratory limits were obtained from subjects not associ- ated with hospital employment or hospital visitation.

Nerve conduction data from AIDP patients included only that obtained during the acute or progressive phase, defined as the first 4 weeks after onset of symptoms. ${ }^{1}$ For CIDP and MND patients, data were analyzed from the initial evaluation in our clinic and, for DP patients, from the initial evaluation in the EMG Laboratory.

Statistical comparisons for differences of proportions for the AMNS pattern between patient groups were made by chi-square tests. Comparisons of group age, sensory nerve response amplitudes, and sural minus median amplitude remainder values were made by analysis of variance using the Fisher protected least-square difference test to make pairwise comparisons at an overall significance level of $P<0.05{ }^{14}$

\section{RESULTS}

The ranges and mean ages of the patient groups are shown in Table 1. Patients in the AIDP group were significantly younger than those in the other groups, and the MND patients were significantly older than the AIDP, CIDP, and DP-study patients $(P<0.05)$.

In the AIDP patient group, the initial study was performed a mean of 2.8 weeks from the onset of symptoms (range $0.6-4.0$ weeks). At that time, the AMNS response pattern was the most common finding (39\%), while the opposite pattern of normal median and abnormal sural responses was rare $(3 \%)$ (Table 1). Because the magnitude of nerve conduction abnormalities evolves during the progressive phase of AIDP and reaches a nadir within 4 weeks, ${ }^{1}$ the robustness of these early findings was investigated. Sixteen patients had a second nerve conduction study within the 4-week period. During the interval, 1 median and 2 sural

Table 1. Demographic data and frequency of occurrence of median and sural sensory nerve response patterns.

\begin{tabular}{|c|c|c|c|c|c|c|}
\hline & $\begin{array}{c}\text { AIDP } \\
(n=36)\end{array}$ & $\begin{array}{c}\text { CIDP } \\
(n=64)\end{array}$ & $\begin{array}{l}\text { DP-consecutive } \\
\quad(n=29)\end{array}$ & $\begin{array}{l}\text { DP-study } \\
(n=30)\end{array}$ & $\begin{array}{c}\text { MND } \\
(n=22)\end{array}$ & $\begin{array}{c}\text { Control } \\
(n=105)\end{array}$ \\
\hline Age (years), mean (range) & $37(3-82)$ & $48(4-71)$ & $57(22-84)$ & $51(22-64)$ & $65(49-82)$ & $41(23-66)$ \\
\hline Both normal & $10(28 \%)$ & $2(3 \%)$ & $0(0 \%)$ & $8(27 \%)$ & $14(55 \%)$ & $100(95 \%)$ \\
\hline Both abnormal & $10(28 \%)$ & $42(66 \%)$ & $23(79 \%)$ & $12(40 \%)$ & $1(5 \%)$ & $1(1 \%)$ \\
\hline Abnormal median, normal sural & $14(39 \%)$ & $18(28 \%)$ & $4(14 \%)$ & $7(23 \%)$ & $5(22 \%)$ & $3(3 \%)$ \\
\hline Normal median, abnormal sural & $2(3 \%)$ & $2(7 \%)$ & $3(10 \%)$ & $4(18 \%)$ & $2(6 \%)$ & $1(1 \%)$ \\
\hline Both absent & $4(11 \%)$ & $10(16 \%)$ & $1(3 \%)$ & $0(0 \%)$ & $0(0 \%)$ & $0(0 \%)$ \\
\hline Median absent, sural present & $7(19 \%)$ & $10(16 \%)$ & $0(0 \%)$ & $0(0 \%)$ & $0(0 \%)$ & $0(0 \%)$ \\
\hline Median present, sural absent & $1(3 \%)$ & $15(23 \%)$ & $13(45 \%)$ & $4(13 \%)$ & $1(5 \%)$ & $0(0 \%)$ \\
\hline
\end{tabular}


responses changed from normal to abnormal, which resulted in 1 less patient classified with the AMNS pattern (36\%).

In the CIDP group, the AMNS response pattern occurred in $28 \%$ (Table 1), which was not significantly different than in the AIDP group $(P=$ $0.27)$. The most common sensory nerve pattern in CIDP patients was for both responses to be abnormal $(66 \%)$.

Among the DP patients (Table 1), the AMNS response pattern occurred less commonly in the DP-consecutive patient group (14\%) than in the DP-study patient group (23\%), but the difference was not significant $(P=0.34)$. In the MND patient group, a surprising $22 \%$ had an AMNS response pattern (Table 1). However, both nerves were normal more often $(55 \%)$ than in any other patient group.

When the same strict criteria were applied to an asymptomatic control group, $5 \%$ had median sensory or sural nerve abnormalities, including $3 \%$ with the AMNS response pattern (Table 1). All abnormalities were just beyond the laboratory limits.

The data were reviewed with attention to the amplitude of the evoked responses. An extreme version of the AMNS pattern, an absent median response and a present sural response, was encountered only in demyelinating polyneuropathy (Table 1). When analyzed quantitatively (Table 2), the lowest average median nerve amplitude occurred in the CIDP group (significantly lower than in all groups except the AIDP group) followed by the AIDP group. Among the patient groups, the largest average median amplitude occurred in the MND group (significantly larger than in all other groups). The average sural nerve amplitude was greater in the AIDP group than in the other patient groups (significantly greater than in all other groups except the MND group). The lowest average sural amplitude occurred in the DP-consecutive patient group (significantly lower than in all other patient groups except the CIDP group).

The age ranges of the MND and control groups were significantly different, and a separate comparison was made between the MND patient group and a control group from another study ${ }^{2}$ with similar mean age and range. The averaged measures of amplitude, distal latency, and conduction velocity were similar for the two groups (data not shown).

The relationship between median and sural nerve amplitudes was investigated. Comparison of median-to-sural amplitude ratios for individual patients was complicated by absent nerve responses which place a zero in the ratio. For purposes of description, but not statistical testing, the median-to-sural amplitude ratio was calculated from the averaged patient group values (Table 2). This ratio of means had a value of 1.0 only for the AIDP group, and higher values in all other patient groups and control subjects. To manage the problem of zero values statistically, the sural amplitude was subtracted from the median amplitude for each patient and the remainders averaged for each group (Table 2). Statistical comparisons revealed that the low remainder values for the AIDP and CIDP patient groups were each significantly different from the remainders in all other patient and control groups $(P<0.05)$, but not different from each other.

A median entrapment mononeuropathy at the wrist could have contributed to the AMNS re-

Table 2. Median and sural sensory nerve response values, mean ratios, and amplitude differences

(mean values and standard errors)

\begin{tabular}{|c|c|c|c|c|c|c|}
\hline & $\begin{array}{c}\text { AIDP } \\
(n=36)\end{array}$ & $\begin{array}{c}\text { CIDP } \\
(n=69)\end{array}$ & $\begin{array}{c}\text { DP-consecutive } \\
\quad(n=29)\end{array}$ & $\begin{array}{l}\text { DP-study } \\
(n=30)\end{array}$ & $\begin{array}{c}\text { MND } \\
(n=22)\end{array}$ & $\begin{array}{c}\text { Control } \\
(n=105)\end{array}$ \\
\hline \multicolumn{7}{|l|}{ Median nerve } \\
\hline Amplitude $(\mu \vee)(\geq 16)$ & $12.9(2.8)$ & $8.6(1.0)$ & $14.1(1.4)$ & $17.4(1.9)$ & $27.1(3.3)$ & $32.7(1.1)$ \\
\hline Distal latency (msec) $(\leq 3.7)$ & $4.2(1.8)$ & $4.2(0.2)$ & $4.0(0.1)$ & $3.0(0.1)$ & $3.4(0.1)$ & $3.0(0.1)$ \\
\hline Conduction velocity $(\mathrm{m} / \mathrm{s})(\geq 53)$ & $57.8(1.9)$ & $48.0(1.6)$ & $45.9(1.3)$ & $53.3(0.9)$ & $55.8(1.6)$ & $60.2(0.5)$ \\
\hline \multicolumn{7}{|l|}{ Sural nerve } \\
\hline Amplitude $(\mu V)(\geq 6)$ & $13.1(1.4)$ & $5.2(0.7)$ & $3.1(0.8)$ & $7.2(0.8)$ & $10.5(1.2)$ & $17.5(0.8)$ \\
\hline Distal latency (msec) $(\leq 4.2)$ & $3.7(0.5)$ & $4.1(0.1)$ & $4.3(0.1)$ & $3.3(0.1)$ & $3.9(0.2)$ & $3.4(0.1)$ \\
\hline Conduction velocity $(\mathrm{m} / \mathrm{s})(\geq 41)$ & $52.3(2.0)$ & $43.9(1.9)$ & $40.7(1.2)$ & $43.2(1.0)$ & $47.1(1.8)$ & $52.2(0.5)$ \\
\hline Median-to-sural amplitude ratio & 0.98 & 1.7 & 4.6 & 2.4 & 2.6 & 1.9 \\
\hline Median-minus-sural amplitude & $-0.1(2.4)$ & $3.4(1.1)$ & $11.0(1.3)$ & $10.2(1.8)$ & $16.6(3.1)$ & $15.2(0.9)$ \\
\hline
\end{tabular}


sponse pattern. A reduced median evoked amplitude may occur early in entrapment neuropathy, but is a common source of false-positive errors. ${ }^{21}$ Accordingly, the median nerve conduction data were reviewed to determine if the AMNS pattern in any patient was based on a prolonged distal sensory latency. If so, the available ulnar sensory nerve data were reviewed to determine if they supported a median mononeuropathy. Among the patients in the AIDP, DP-consecutive, and DPstudy groups, none had the AMNS response pattern due to an isolated abnormality of median distal latency. One CIDP patient and 1 MND patient had only prolonged median distal latencies and no ulnar conduction studies for clarification and may have had median mononeuropathies at the wrist. In the control group, 1 subject with the AMNS response pattern may have had a median mononeuropathy.

\section{DISCUSSION}

The AMNS response pattern occurred most frequently in demyelinating polyneuropathy, with sensitivity (true-positive rate) of $39 \%$ in AIDP patients during the first 4 weeks of symptoms and 28\% in CIDP patients. The specificity (true negative rate) of this pattern in other peripheral nerve disorders was $77-86 \%$ in DP patient groups and $78 \%$ in MND patients. The pathophysiology of the AMNS pattern in AIDP may be explained by early demyelination of small caliber sensory nerve fibers causing slowing and conduction block along distal segments. The recording of this pattern is based on the position of ring recording electrodes on digital branches of the median sensory nerve compared to disc recording electrodes on the sural nerve trunk at the level of the lateral malleolus $^{3}$ (Fig. 1). This can explain the findings of low average median nerve amplitude values, low ratio of median-to-sural average amplitude values, and low average differences between median and sural amplitudes in the AIDP patient group compared with other groups. There is supportive evidence from motor nerve conduction studies in AIDP for early and severe demyelinating changes occurring in very distal or terminal branches. $1,18,19$

With the prolonged course of CIDP, pathologic changes progress to include more proximal portions of nerve. ${ }^{22}$ This can explain the finding, in CIDP patients, of a lower percentage with the AMNS pattern; a greater percentage with abnormalities of both median and sural nerves; and lower average median- and sural-evoked amplitudes compared with AIDP patients.
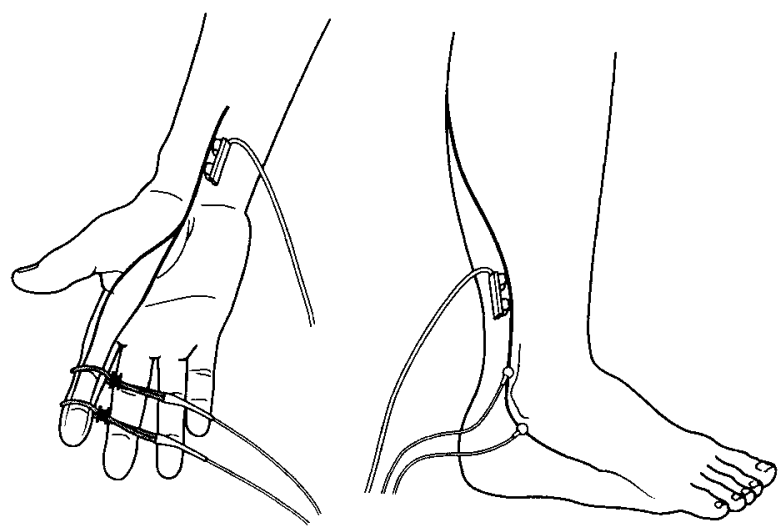

FIGURE 1. Diagram showing the location of recording electrodes to highlight the different electrode positions along the distal segments of the median sensory nerve (left) and sural nerve (right).

The AMNS response pattern was recorded less frequently in patients with neuropathic disorders in which axonal involvement predominates or in which sensory nerve involvement is minimal. The 2 groups of diabetic patients were presented separately because the DP-study patient group represents mild polyneuropathy and the DP-consecutive patient groups represents more severe polyneuropathy, as reflected in more abnormal averaged measures in the latter group (Table 2). The more common occurrence of the AMNS response pattern in the DP-study patients can be explained by early involvement of distal nerve segments (digital nerves), and the less common occurrence of the AMNS pattern in DP-consecutive patients by later involvement of more proximal segments (sural nerve at the ankle). A distal-to-proximal progression of nerve involvement sufficient to affect the AMNS pattern was not observed in AIDP patients during the 4-week active phase.

The presence of the AMNS response pattern in 3 control subjects likely reflects two factors. One is the effect of setting the EMG Laboratory normal limits at 2 standard deviations. The other is the effect of age on nerve conduction values, for 2 of the 3 with the AMNS pattern were older (53 and 61 years).

The AMNS response pattern in MND patients can be attributed to several factors. One is agerelated changes, as discussed above. A second factor is the likely presence of a mild axonal sensory neuropathy in MND, which is supported by histo$\operatorname{logic}^{5,13}$ and electrophysiologic data. ${ }^{4,9}$ A third factor is difficulty achieving adequate temperature control of atrophic digits and limbs despite ade- 
quate warming. ${ }^{4}$ These factors can affect median more than sural responses because of the different recording electrode placements. The dominant findings in the MND patient group is that they have the greatest percentage of both nerves normal and the lowest percentage of both nerves abnormal (Table 1).

A comparison can be made between the diagnostic sensitivity of motor and sensory nerve conduction abnormalities for primary demyelinating polyneuropathy. Support for primary demyelination from motor nerves can be based on fulfillment of formal sets of electrodiagnostic criteria, ${ }^{3}$ and sensitivity has been found to reach $85 \%$ in AIDP and $64 \%$ in CIDP, and specificity $100 \%$ for DP-consecutive and MND groups. ${ }^{1,6}$ The AMNS sensory nerve response pattern is, therefore, less sensitive and less specific for demyelinating polyneuropathy. However, two other sensory nerve patterns are more specific for demyelinating polyneuropathy; the average median-to-sural amplitude ratio was 1.0 only in the AIDP group, and very low remainder values for the difference between median and sural amplitudes were found only in AIDP and CIDP patients. Accordingly, in the setting of appropriate motor nerve abnormalities and clinical features, the pattern of an AMNS response, a low median-to-sural amplitude ratio, or a minimal difference between amplitude values is highly supportive of the diagnosis of a primary demyelinating polyneuropathy and against a primary axonal polyneuropathy.

\section{REFERENCES}

1. Albers JW, Donofrio PD, McGonagle TK: Sequential electrodiagnostic abnormalities in acute inflammatory demyelinating polyradiculoneuropathy. Muscle Nerve 1985;8: $528-539$.

2. Albers JW, Kallenbach LR, Fine LJ, Langolf GD, Wolfe RA, Donofrio PD, Alessi AG, Stolp-Smith KA, Bromberg MB: Neurological abnormalities associated with remote occupational elemental mercury exposure. Ann Neurot $1988 ; 24: 651-659$.

3. Albers JW, Kelly JJ: Acquired inflammatory demyelinating polyneuropathies: clinical and clectrodiagnostic features. Muscle Nerce 1989;12:435-451.
4. Behnia M, Kelly JJ: Role of electromyography in amytrophic lateral sclerosis. Muscle Nerve 1991;14:12361241 .

5. Bradley WG, Good P, Rasool CG, Adelman IS: Morphometric and biochemical studies of peripheral nerves in amyotrophic lateral sclerosis. Ann Neurol 1983;14:267277.

6. Bromberg MB: Comparison of clectrodiagnostic criteria for primary demyelination in chronic polyneuropathy Muscle Nerue 1991;14:968-976.

7. Bromberg $\mathrm{MB}$, Albers JW: Nerve conduction studies in chronic and acute inflammatory demyelinating polyradiculoneuropathies. Muscle Nerve 1988;1 1:960 (abstract).

8. Brown MJ, Asbury AK: Diabetic neuropathy. Ann Neurol 1984;15:2-12.

9. Brown WF, Jaatoul N: Amyotrophic lateral sclerosis. Electrophysiologic study (number of motor units and rate of decay of motor units). Arch Neurol 1974:30:242-248.

10. Burke D, Skuse NF, Lethlean K: Sensory conduction of the sural nerve in polyneuropathy. I Neurol Neurosurg Psy chiatry 1974;37:647-652.

11. Donofrio PD, Albers JW: Polyneuropathy: classification by nerve conduction studies and electromyography. Muscle Nerve 1990; 13:889-903.

12. Dyck PJ, Lais AC, Ohta M, Bastron JA, Okazaki H, Groover RV: Chronic inflammatory polyradiculoneuropathy. Mayo Clin Proc 1975;50:621-637.

13. Dyck PJ, Stevens JCC, Mulder DW, Espinosa RE: Frequency of nerve fiber degeneration of peripheral motor and sensory neurons in amyotrophic lateral sclerosis. Neurology $1975 ; 25: 781-785$

14. Hochberg Y, Tamhane AC: Multiple Comparison Procedures New York, Wiley, 1987, pp 2-5.

15. Kimura J, Machida M, Ishida T, Yamada 'T, Rodnitzky R, Kudo Y, Suzuki S: Relation between size of compound sensory or muscle action potentials, and length of nerve segment. Neurology 1988;36:647-652.

16. Kimura J: Electrodiagnosis in Diseases of Nerve and Muscle: Principles and Practice (2 Ed). Philadelphia, F.A. Davis, 1989, pp 103-138.

17. Kincaid JC, Minnick KA, Pappas S: $\Lambda$ model of the differing change in motor and scnsory action potentials over distance. Muscle Nerve 1988;11:318-323.

18. McLeod JG, Walsh JC, Prineas JW, Pollard JD: Acute idiopathic polyneuritis. J Neurol Sci 1976;27:145- 162.

19. Miller RG, Peterson C, Rosenberg NL: Electrophysiologic evidence of severe distal nerve segment pathology in the Guillain-Barré syndrome. Muscle Nerve 1987;10:524-529.

20. Murray NMF, Wade DT: The sural sensory action potential in Guillain-Barré syndrome. Muscle Nerve 1980:3:444 (letter).

21. Redmond MD, Rivner MH: False positive electrodiagnostic tests in carpal tunnel syndrome. Muscle Nerce 1988;11: $511-517$.

22. Wexler 1: Sequence of demyelination-remyelination in Guillain-Barré disease. J Neurol Neurosurg Psychiatry 1983:46:168-174. 